\title{
Rough droplet model for spherical metal clusters
}

\author{
Nicolas Pavloff and Charles Schmit
}

Division de Physique Théorique円, Institut de Physique Nucléaire, F-91406 Orsay Cedex, France

\begin{abstract}
We study the thermally activated oscillations, or capillary waves, of a neutral metal cluster within the liquid drop model. These deformations correspond to a surface roughness which we characterize by a single parameter $\Delta$. We derive a simple analytic approximate expression determining $\Delta$ as a function of temperature and cluster size. We then estimate the induced effects on shell structure by means of a periodic orbit analysis and compare with recent data for shell energy of sodium clusters in the size range $50<N<250$. A small surface roughness $\Delta \simeq 0.6 \AA$ is seen to give a reasonable account of the decrease of amplitude of the shell structure observed in experiment. Moreover - contrary to usual Jahn-Teller type of deformations - roughness correctly reproduces the shape of the shell energy in the domain of sizes considered in experiment.
\end{abstract}

PACS numbers :

36.40.+d Atomic and molecular clusters.

71.20.-b Electron density of states.

03.65.Sq Semiclassical theories and applications.

IPNO/TH 97-19 to appear in Phys. Rev. B

\footnotetext{
${ }^{1}$ Unité de Recherche des Universités Paris XI et Paris VI associée au CNRS.
} 


\section{Introduction}

Since the discovery of shell effects in metal clusters, the mean field approach with delocalized electrons has been a very efficient tool for describing a wide variety of phenomena : shell and supershell effects, dipole polarizability and optical excitations, fission ... (for reviews see Refs. [1, 2]).

This type of approach mainly relies on the jellium model where the ionic background is considered as a smooth and uniform distribution of positive charges. It is best legitimate for simple metals (and to a lesser extend for noble metals) with delocalized valence electrons, almost insensitive to the actual arrangement of the ionic cores. Hence the best candidates for this approximation are the alkali metals, as can be inferred from the quasi-sphericity of their Fermi surface (revealing a weak interaction between ionic cores and valence electrons). As a result the compressibility of these solids is close to its electron gaz value, and the surface tension is correctly described by the jellium models (the agreement being better for small electronic density, see Ref. [3]).

It was realized early that deformation effects had to be taken into account for a realistic des-

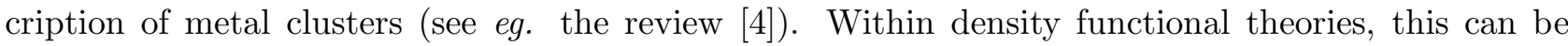
achieved by imposing to the jellium the shape that suits the electrons best [5 [7]. Deformations have also been studied in less elaborate models (deformed external mean-field) [8] 13] and there is a good overall agreement with experimental data for ionization potentials, dissociation energies and splitting of dipole resonances for relatively small clusters (less than 40 atoms).

The above mentioned deformations are of Jahn-Teller type and occur between major shell closures, where lowering the symmetry leads to a gain in energy. Another type of surface deformation has also to be considered which consists in surface irregularities of very large multipolarities. These deformations not only lower the shell effect but also introduce randomness into the spectrum. This was first noticed by Gor'kov and Eliashberg [14] who claim that "the distribution of the levels should be random even if the particles have the same volume and a good shape, say spherical particles of equal size. The point is that electrons in the metal have a wavelength of the order of atomic dimensions. Therefore surface irregularities of atomic size are sufficient to make the level distribution perfectly random". This statement has to be tempered in view of the success of the jellium model. Nevertheless it makes no doubt that the surface of a cluster has atomic size irregularities, it is important to estimate their amplitude and to evaluate the resulting effect on the physical observables.

The fact that disorder is located on the surface is legitimated because the elastic mean free path of an electron in the bulk is typically of order of several hundreds of Angströms, whereas an electron experience collisions on the surface of the cluster about each $10 \AA$. Indeed it was shown in Ref. [15] that the scattering of electrons on the fluctuation of the positive ions had an effect of the same order as that of thermal distribution of occupancy probability ; which in turn is shown in the present work to be negligeable compared to the effect of shape fluctuations. More microscopically, bulk disorder would be represented by fluctuations of the bottom of the potential well and in the large size limit high lying states tend to be insensitive to this perturbation, whereas the effects of surface disorder increase when one goes up in the spectrum [16]. Note that such irregularities are to be taken into account also when the cluster is "liquid-like" : the mean velocity of the ionic cores is always by several orders of magnitude smaller than the typical electronic Fermi velocity. Hence, as far as electronic motion is 
concerned, the ionic cores can be considered as frozen and this necessarily implies a certain degree of surface roughness.

In the present paper we use a liquid drop model to study the thermally activated surface deformations, or capillary waves, of a neutral spherical cluster (Sec. 2). These deformations correspond to a surface roughness which we characterize by a single parameter $\Delta$. We derive a simple analytic approximate expression determining the behaviour of $\Delta$ as a function of temperature and cluster size. Then in Section 3 we discuss the influence of shape fluctuations on the level density using a trace formula in rough billiards and compare with thermal effects linked with Fermi occupation number of the energy levels (for this purpose we give the general form of shell corrections in the presence of temperature in Appendix C). Finally we present our conclusions and discuss possible refinements of our approach in Sec. 4.

\section{Liquid drop model}

In the liquid drop model, a cluster is described as a droplet of incompressible fluid whose shape can be parameterized by a set of normal coordinates $\alpha_{\lambda \mu}$ obtained by expanding the surface in spherical harmonics [17, 18]:

$$
r(\Omega, t)=R\left[1+\sum_{\lambda \mu} \alpha_{\lambda \mu}(t) Y_{\lambda \mu}(\Omega)\right] .
$$

The r.h.s. of Eq. (11) is made real by imposing $\alpha_{\lambda,-\mu}=(-)^{\mu} \alpha_{\lambda \mu}^{*}$. The summation stops at a Debye cutoff $\Lambda$ estimated by equating the number of surface modes to the number of atoms on the surface, this yields $\Lambda=(3 \sqrt{4 \pi} N)^{1 / 3} \simeq 2.20 N^{1 / 3}$. The droplet being considered as incompressible one should impose volume conservation. If the cluster contains $N$ atoms it should have a volume $V=4 \pi R^{3} / 3$ with $R=r_{S} N^{1 / 3}$ ( $r_{S}$ being the Wigner-Seitz radius of the material). This leads to the relation $\alpha_{00} \sqrt{4 \pi}=-\sum_{\lambda \mu}\left|\alpha_{\lambda \mu}\right|^{2}$, valid to leading order. Also the modes $\lambda=1$ which correspond to a global translation of the drop should be omitted in the summation (11).

Eq. (11) yields a kinetic energy $T$ and a surface energy $V_{\text {surf }}=\sigma \mathcal{A}$, where $\sigma$ is the surface tension and $\mathcal{A}$ the surface area corresponding to (1). Including terms up to second order in the $\alpha$ 's one obtains [17, 18]:

$$
T=\frac{\rho_{0} R^{5}}{2} \sum_{\lambda \mu} \frac{\left|\dot{\alpha}_{\lambda \mu}\right|^{2}}{\lambda} \quad \text { and } \quad V_{\text {surf }}=4 \pi R^{2} \sigma+\frac{R^{2} \sigma}{2} \sum_{\lambda \mu}\left|\alpha_{\lambda \mu}\right|^{2}(\lambda-1)(\lambda+2) .
$$

where $\rho_{0}$ is the specific mass of the material considered.

One can also take into account a curvature term in the potential energy

$$
V_{\text {curv }}=\frac{\gamma}{4} \int d A\left(\frac{1}{\mathcal{R}_{1}}+\frac{1}{\mathcal{R}_{2}}\right) .
$$

In (3) $\gamma$ is an intrinsic curvature energy parameter, $\mathcal{R}_{1}$ and $\mathcal{R}_{2}$ are the principal radii of curvature. It turns out (see Appendix A) that taking this term into account exactly amounts to replacing in Eq. (2) the surface tension by an effective term $\sigma \rightarrow \sigma^{*}=\sigma+\gamma /(2 R)$ with which we will work henceforth. 
No other contribution to the potential energy has to be taken into account because we consider concomitant deformations of the jellium and of the valence electron cloud of a neutral cluster (hence there is no other electrostatic deformation energy than the one included in (2,3)). We neglect at this level finite size quantum effects. We use for the surface tension $\sigma$ the value of the bulk material extracted from experiment in Ref. [19], and this implicitly contains quantal effects associated with the kinetic energy of the electrons near the surface. Hence including quantum effects in the present description would double count this contribution ; the appropriate procedure would be to use a Strutinsky shell correction, $c f$. the discussion at the end of the paper. In Ref. [20] a description analogous to the present one has been shown to account accurately of the monovacancy formation energy in simple metals such as the one we are interested in. This gives us confidence in the ability of a liquid drop model to describe atomic size irregularities. Note that in [20] the value of $\sigma$ is renormalized in order to describe an ideally flat surface. This procedure should not be employed here because we want the surface tension of a large cluster to tend to the one of the bulk material.

Eqs. (2) and (3) correspond to a liquid drop Lagrangian $\mathcal{L}_{L D}\left[\dot{\alpha}_{\lambda \mu}, \alpha_{\lambda \mu}\right]=T-V_{\text {surf }}-V_{\text {curv }}$ with normal modes $\alpha_{\lambda \mu}(t)=A_{\lambda \mu} \exp \left(i \omega_{\lambda} t\right)$ of pulsation $\omega_{\lambda}$ given by :

$$
\omega_{\lambda}^{2}=\lambda(\lambda-1)(\lambda+2) \frac{\sigma^{*}}{\rho_{0} R^{3}}
$$

and the classical energy of the mode is $E_{\lambda \mu}=\sigma^{*} R^{2}(\lambda-1)(\lambda+2)\left|A_{\lambda \mu}\right|^{2}$. The average value of the amplitude $\left|A_{\lambda \mu}\right|^{2}$ of the thermally activated mode is determined by writing $E_{\lambda \mu}=k_{B} T$. We use here classical statistical mechanics, the quantal analog would be $E_{\lambda \mu}=\hbar \omega_{\lambda}\left(n_{\lambda}+1 / 2\right)$, where $n_{\lambda}=\left[\exp \left(\hbar \omega_{\lambda} / k_{B} T\right)-1\right]^{-1}$ is a Bose occupation factor. Such a description has been used for describing the surface oscillations of liquid helium [21], but here the motion of the surface is classical : $k_{B} T \gg \hbar \omega_{\Lambda}$ (from (4) $\hbar \omega_{\Lambda} \simeq 130 \mathrm{~K}$ for sodium).

From (11) the quantity $r(\Omega, t)$ averaged over the surface has a mean value $R\left(1+\alpha_{00} / \sqrt{4 \pi}\right)$ and a standard deviation $\Delta$ which is is given by $\Delta^{2}=R^{2} \sum_{\lambda \geq 2}\left|\alpha_{\lambda \mu}\right|^{2} /(4 \pi)$, hence $\Delta$ is independent of time. The explicit formula reads :

$$
\Delta^{2}=\frac{k_{B} T}{4 \pi \sigma^{*}} \sum_{\lambda=2}^{\Lambda} \frac{2 \lambda+1}{(\lambda-1)(\lambda+2)} \simeq \frac{k_{B} T}{4 \pi \sigma^{*}} \ln \frac{(2 \Lambda-1)(2 \Lambda+5)}{7} .
$$

In the r.h.s. of Eq. (5) we replaced the discrete summation by the first term of its Euler-MacLaurin expansion. Figure (1a) displays the result of Eq. (可) for sodium clusters at temperatures $T=200 \mathrm{~K}$ and $T=450 \mathrm{~K}$ in the size region $20 \leq N \leq 1000$. It is difficult to determine the precise value of $\sigma^{*}$ to be used in Eq. (5) : the surface and curvature parameters $\sigma$ and $\gamma$ depend on temperature and of the actual phase (liquid or solid) of the aggregate. Hence we used several values of $\sigma$ and $\gamma$ : a lower bound for $\Delta$ is obtained by taking the values $\sigma=190 \mathrm{~K} . \AA^{-2}$ (which is the solid-vapor value extrapolated to zero temperature in Ref. [19]) and $\gamma=285 \mathrm{~K} . \AA^{-1}$ [20]. The upper bound is obtained by taking $\gamma=0$ and $\sigma=145 \mathrm{~K} . \AA^{-2}$ (which is the liquid-vapor surface tension at melting [19]). These values of $\sigma$ correspond to a droplet parameter $a_{s}=4 \pi r_{S}^{2} \sigma$ which ranges from $0.68 \mathrm{eV}$ (for $\sigma=145$ $\mathrm{K} . \AA^{-2}$ ) to $0.89 \mathrm{eV}$ (for $\sigma=190 \mathrm{~K} . \AA^{-2}$ ). Indeed one can find a large dispersion of $a_{s}$ in the literature : the value $0.54 \mathrm{eV}$ was used in Refs. [12,22] (from a fit to theoretical values of clusters' energy) ; in 
Ref. [2] the value $a_{s}=0.7 \mathrm{eV}$ was extracted from the bulk surface tension and in Ref. 23] the value $a_{s}=1.02 \mathrm{eV}$ was obtained via experimental determination of clusters' cohesive energy.

( a )

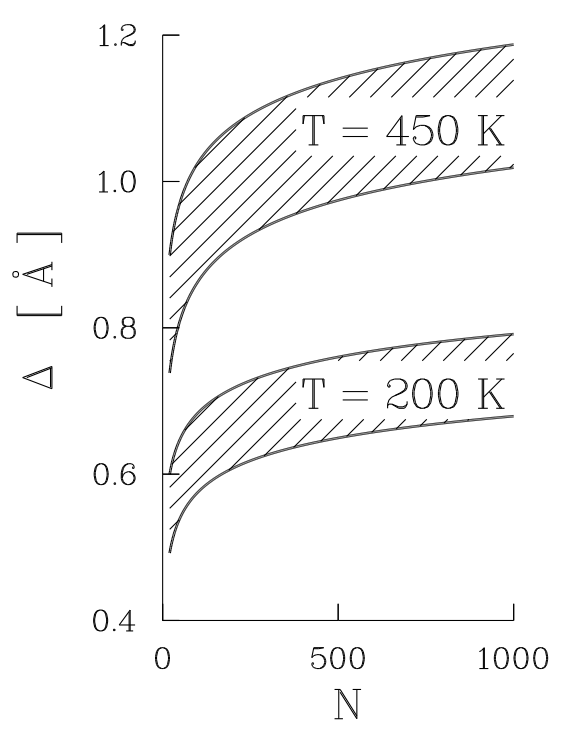

( b )

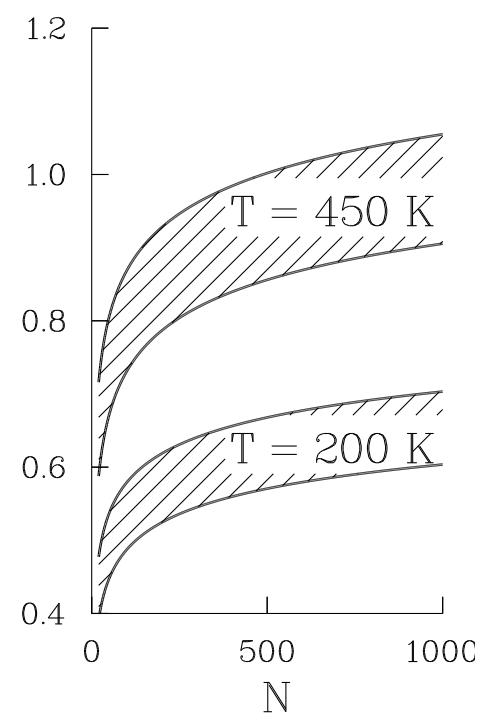

Figure 1: Part (a) : $\Delta$ as a function of $N$ for sodium clusters at temperatures $200 \mathrm{~K}$ and $450 \mathrm{~K}$, from Eq. (5). The shaded zones correspond to different values of the surface tension and curvature parameter (see the text). Part (b) : same as part (a) withdrawing in Eq. (5) the contribution of the two first multipolarities $(\lambda=2$ and $\lambda=3)$.

As several studies in the field have demonstrated (see e.g the work of Yannouleas and Landman [12, 24]) the liquid-drop Lagrangian $\mathcal{L}_{L D}$ is not adequate for describing the deformations of small multipolarities, which are determined mainly by shell effects (see the discussion at the end of the paper). In order to estimate the role of these multipolarities in the amplitude of the roughness we have redrawn Figure (1a) withdrawing the contributions of $\lambda=2$ and 3 in the summation (5). The result is displayed on part (b) of Fig. 1. As one should expect this significantly reduces the value of $\Delta$. For instance, at $T=450 \mathrm{~K}$ the typical roughness for $N \simeq 200$ is reduced from $1 \AA$ to $0.85 \AA$.

From the discussion above and the comparison of Figures (1a) and (1b), we see that due to the simplicity of our model we cannot precisely determine the surface roughness of sodium clusters at typical experimental temperatures. For sodium $r_{S}=2.08 \AA$ (thermal variations of $r_{S}$ are negligeable here), and we can only say that typical roughnesses are of order of 30 to $50 \%$ of the interatomic distance. However it makes no doubt that there is a thermal activation of capillary waves which gives a contribution to the surface roughness of the type given by Eq. (5) for large enough multipolarities. 
We will see below that this has an important effect on shell structure, and that this effect is crucial for understanding the shape of the shell energy determined in experiment.

Note that an estimation of the geometrical corrugation of a solid surface at zero temperature [20] yields values of $\Delta$ of order of $20 \%$ of $r_{S}$. Hence the reduction of shell oscillations presented below is a general phenomenon which does not depend on the solid or liquid structure of the aggregate.

\section{3 shell energy in a rough sphere}

The influence of surface roughness on the level statistics has been discussed in Refs. 25 28] and more recently in a 2D model [29]. In the present work we concentrate on its effect on level density and shell structure. Similar effects have been recently studied in Refs. [30, 31] and [32]. The spirit of the present section is very similar to the one of Ref. [32] which presents numerical results in a corrugated mean field. However the qualitative conclusions are different : in Ref. [32] corrugation is seen to imply a shift in the supershell structure. This effect - not seen in the present study - seems to be due to the fact that, in a finite depth potential such as the one used in [32], roughness leads to an effective mean-field where the phase difference between the orbits is modified. In any case, for the small roughnesses we are using here, the shift in the shell structure found in Ref. [32] is small. In Ref. [30] the disorder is modelized by the addition of a random Hamiltonian to the mean field and the results are comparable to the one presented below. The approach has been further extended in Ref. [31] where the effects of disorder on energetics of lithium, sodium and potassium clusters where taken into account in a liquid drop plus shell-correction model. Here the Hamiltonian for the deformation is only of liquid drop type ; however we do take into account the thermally activated oscillations for the Hamiltonian we consider (see the discussion in Sec. 4).

In the present work the $N$ electrons are considered to be moving independently in an infinite potential well (a billiard) having a shape approximatively spherical (as given by (1D)). Hence we can consider that the actual shape is obtained by a random deviation from a perfect sphere, with Gaussian fluctuations of standard deviation $\Delta$ determined above. The choice of Gaussian fluctuations reflects the fact that the distribution of each $A_{\lambda \mu}$ is Gaussian (according to classical mechanics). Then invoking the central limit theorem it can reasonably be considered that the shape fluctuations are of Gaussian type. We consider an ensemble of clusters (such as one would expect in a molecular beam) and we will present results averaged over this ensemble. The radius $R$ of the average sphere scales with $N$ so that the mean electronic density is kept constant and equal to its bulk value : $R=r_{S} N^{1 / 3}$.

The level density in a rough billiard with small size surface irregularities was studied in Ref. [16] and a semiclassical trace formula averaged over surface disorder was derived. The important feature of the level density is the gradual disappearance of shell effects with increasing energy : near the Fermi level the electronic wavelength is of order of the typical size of the surface defects and the induced shift of the eigenlevels leads after averaging to a structureless level density. The bottom of the spectrum is not affected because low lying state have a wavelength much larger than the surface perturbations (accordingly, the effect on level statistics is different at the bottom of the spectrum and near the Fermi energy [27]).

It is shown in Ref. [16] that the oscillatory part of the electronic energy $E_{\text {shell }}$ (the so-called shell 
energy) can be expressed on average as a sum over classical periodic orbits in a perfect sphere :

$$
E_{\text {shell }}(N, \Delta) \simeq \frac{\hbar^{2} \bar{k}_{F}^{2}}{2 m} \sum_{P O} \frac{2 \mathcal{A}\left(\bar{k}_{F}\right)}{\bar{k}_{F} L^{2}} \sin \left(\bar{k}_{F} L+\nu \pi / 2\right) \exp \left\{-2 n\left(\bar{k}_{F} \Delta\right)^{2} \cos ^{2} \theta\right\} .
$$

In (6) $m$ is the electron mass, $\bar{k}_{F}$ is the smooth part (i.e. non-oscillatory) of the Fermi wave-vector, which is to a good approximation equal to the bulk wave-vector $\kappa_{F}=r_{S}^{-1}(9 \pi / 4)^{1 / 3}$. $E_{\text {shell }}$ in (6i) is a quantity averaged over surface disorder, but the sum is performed over all the periodic orbits (POs) of a perfect sphere (see [16]). $L$ is the length of a PO, $\mathcal{A}$ is an amplitude slowly depending on $\bar{k}_{F}, \nu$ is a Maslov index, $n$ is the number of bounces of the PO on the sphere and $\theta$ is the bouncing angle ; all these quantities depend on the PO considered, see Appendix B for further details. When $\Delta=0$, (6) follows from Balian and Bloch's trace formula for the sphere [33]. Since $\bar{k}_{F}$ is nearly constant, the main $N$-dependence in (6) is due to the scaling of the cluster's size according to $R=r_{S} N^{1 / 3}$ ( $L$ scales like $R, \mathcal{A} \propto R^{5 / 2}$ or $R^{2}$ for some orbits, see Appendix A).
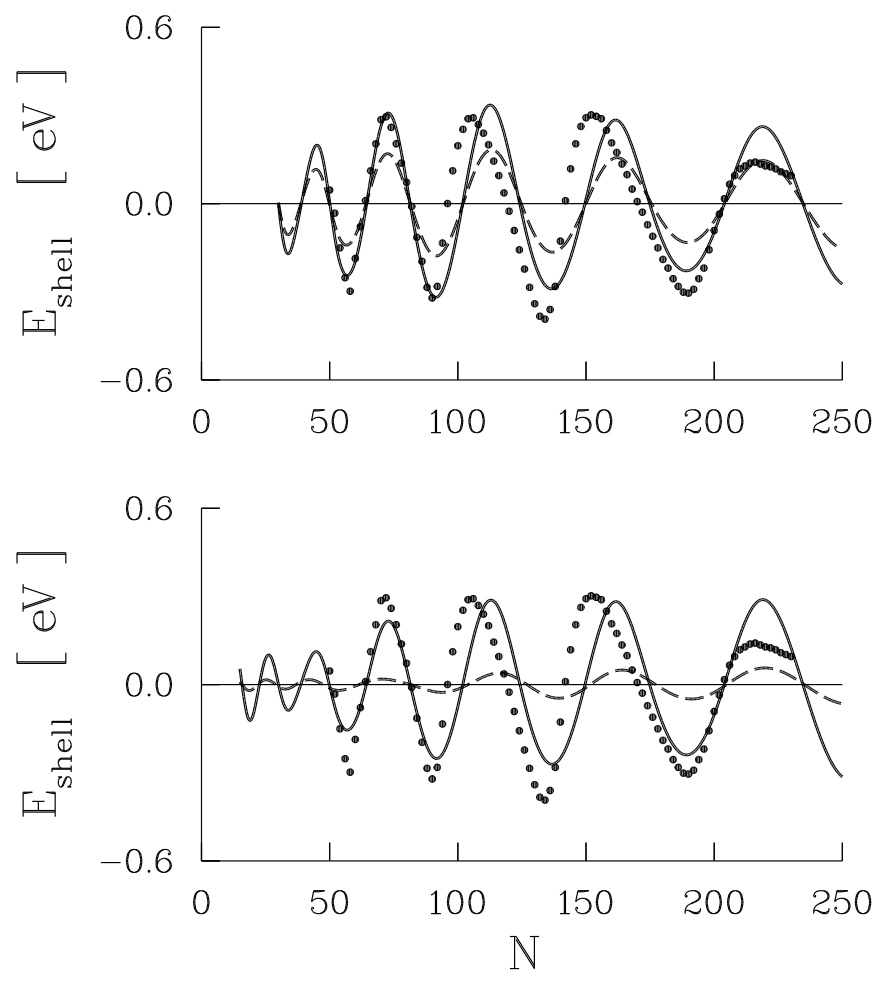

Figure 2: $E_{\text {shell }}(N, \Delta)$ as a function of $N$ in sodium clusters. The lower plot compares the experimental results of Ref. [34] (black points) with the values obtained by fixing $\Delta=0.9 \AA$ (dashed line) and $\Delta=0.63 \AA$ (solid line). The upper plot compares the experimental data with the values obtained by determining for each cluster size $\Delta$ via (5), taking $\sigma=190 \mathrm{~K} . \AA^{-2}$ and $\gamma=285 \mathrm{~K} . \AA^{-1}$, withdrawing the contributions of $\lambda=2$ and 3 . The dashed line corresponds to a temperature $T=400 \mathrm{~K}$ and the solid line to $T=300 \mathrm{~K}$. 
$E_{\text {shell }}$ as given by (6) is compared on Fig. 2 with the value determined by Chandezon et al. in Ref. [34. In this reference an evaporation model is used for extracting the shell energy from the abundance distribution in a beam and results for clusters of sizes ranging from 50 to 230 atoms are obtained. This experiment is important for our study because it concerns relatively large clusters and our approach is limited to this domain for the two following reasons : (i) we use a semiclassical approach more accurate for large sizes (see for instance the comparison with exact results on Fig. 4) and moreover (ii) the macroscopic concept of roughness is meaningless for small sizes ; for instance in a cluster with $N=20$ atoms the Debye cut-off fixes the maximum angular momentum of surface deformation to be $\Lambda \simeq 2.2 N^{1 / 3} \simeq 6$ and in this regime the concept of roughness is of marginal importance (see below the discussion of Jahn-Teller effect). In the following we state rather loosely that our approach is relevant for sizes $N \gtrsim 100$.

On the lower part of Fig. 2 the dashed curve corresponds to a constant value $\Delta=0.9 \AA$ (independent of $N$ ) which - from Fig. 1 - is a typical value at $T=450 \mathrm{~K}$ (this temperature is in agreement with usual evaporation conditions [35 37]). The fact that this value of $\Delta$ leads to a too large damping of shell structure should not worry us at this level : as stated in the previous section, the liquid drop model does not accurately determine the value of $\Delta$ because it does not properly describe deformations of small multipolarities. These deformations should be described with a more elaborate procedure and may be less easily thermally excited (see the discussion of Sec. 4). This is confirmed by the solid curved which is drawn for $\Delta=0.63 \AA$ and which gives a better account of the data. Note the sensitivity of $E_{\text {shell }}$ to the value of $\Delta / r_{S}$ : formula (6) has a schematic large $N$ behaviour of the form :

$$
E_{\text {shell }}(N, \Delta) \sim \varepsilon_{F} N^{1 / 6} \exp \left(-\Delta^{2} / r_{S}^{2}\right) \sin \left(N^{1 / 3}\right) .
$$

where $\varepsilon_{F}=\hbar^{2} \kappa_{F}^{2} /(2 m)$ is the bulk Fermi energy. For clarity we have dropped in the sine and the exponent of (7) important but dimensionless factors. This will be done also in (9) ; the derivation of these formulae is explained in Appendix C (Eq. (C9)). Hence the shell structure is very sensitive to a small surface roughness ; this point and the validity of formulae of type (6) have been further tested on a numerical example in Ref. [38].

The value $\Delta=0.63 \AA$ does not quite correspond to the estimation of Fig. 1 for $T=450 \mathrm{~K}$ : as explained in the conclusion the liquid drop Lagrangian seems to underestimate the stiffness of the potential for the deformation parameters. It may also happen that the electrons experience a meanfield which - due to the diffuseness of its surface - is less corrugated than the ionic background. In the same line, instead of fixing $\Delta$ to a constant value, one should according to (5) take size-dependence of the roughness into account. This improves the agreement with experiment for $N \simeq 50$ since in this region the value of $\Delta$ decreases significantly (see Fig. 1) and this leads to lower damping of the theoretical curve which comes closer to experiment. This has been done on the upper part of Fig. 2 which is drawn in the case $\sigma=190 \mathrm{~K} . \AA^{-2}$ and $\gamma=285 \mathrm{~K} . \AA^{-1}$; these values have been chosen because they lead to small values of $\Delta$ and to relatively good agreement with experiment. The dashed line correspond to $T=400 \mathrm{~K}$ and the solid line to $T=300 \mathrm{~K}$. For each temperature and cluster size $\Delta$ was determined via (5), withdrawing the contributions of $\lambda=2$ and 3. However, such a refinement is unnecessary for larger cluster sizes in view of the small $N$-dependence of (5) for large $N$. Besides, the simple model with a constant value of $\Delta=0.63 \AA$ gives already a satisfactory agreement with experimental data. 
In fact our approach is more strongly supported by the very good comparison of theory an experiment for the shape of the curve for the shell energy than for the comparison with the amplitude. Indeed the agreement with the amplitude may not be as good as presented on Fig. 2 because there should be some room left for an extra reduction of the amplitude of the shell energy due do quantum mechanically driven deformations, corresponding to relatively small multipolarities $(\lambda=2$ or 3 typically). Nevertheless, due to the high sensitivity of formula (6) to small changes of $\Delta$, we still can conclude from Fig. 2 that the typical roughness is of order of $0.6 \AA$.

Concerning the shape of the curve, the experimental results of Ref. [34] are surprising because they are in contradiction with the common belief that cluster's deformations are only governed by Jahn-Teller effects. For instance, the dissociation energies and ionization potentials of simple metal clusters of relatively small sizes are well accounted for by models where the Jahn-Teller effect is the only mechanism of deformation [12,24] (the agreement with experiment survives up to size $N \sim 100$ for the ionization potentials of potassium, see Ref. [39]). This phenomenon was expected to occur even for large values of $N$, see e.g. the zero temperature results of Refs. [9] 11, 13], or the finite temperature results of Ref. [40]. In these studies, the deformations occur between shell closure and their main effect is to remove the upper part of the shell oscillations ; the shell energy is predicted to have sharp negative spikes in vicinity of the magic numbers (these spikes correspond semiclassically to long POs). On the other hand surface roughness suppresses long POs and reduces shell structure more uniformly, as seen in the experimental data of Ref. [34]. Hence we feel that previous theoretical approaches overestimate the role of the Jahn-Teller mechanism : the very specific shape of shell energy they predict is not seen in the experiment of Chandezon et al. The comparison between our approach and the experimental results for the sizes $N \gtrsim 100$ firmly establishes that there is a qualitatively important effect of roughness.

On the quantitative level, one can also notice that typical theoretical studies overestimate the shell effect for clusters of large sizes : compare Fig. 3 of Ref. [34] with similar figures of Refs. [9 11, 13]. Temperature effects improve the agreement (see Ref. [40]) but there is still a mismatch of order of 40 $\%$ for the amplitude of shell oscillations (see Fig. 3 of Ref. [34]), leaving room for improvement due to surface roughness.

For further comparison with experimental data we display on Table 1 the magic numbers in the region $N<1300$. The first column shows the $\Delta=0$ results from the semiclassical formula (6). The second column displays the exact result in the perfect sphere and merely tests the accuracy of the semi-classical periodic orbit expansion used in the first column. Note that the magic numbers of these two columns are almost identical to the results of Bulgac and Lewenkopf [10] who use a quadrupole deformation of a spherical billiard model within the shell correction method : this is due to the fact that, as stated above, there is no Jahn-Teller deformation at shell closure. In the third column we show the magic numbers obtained from (6) with $\Delta=0.63 \AA$. The three first columns compare well with the experimental ones from Chandezon et al. (column 4), and roughness has only a small effect on the location of the magic numbers. We still produce these data because they justify the billiard model we are using : the magic numbers from Table 1 are in better agreement with experiment than the one obtained with harmonic oscillators [9, 11] or more elaborate potentials [13, 40]. Hence, as far as the phase difference between the contribution of its POs to (6) is concerned, the billiard model is presumably close to the experimental situation since it allows a good prediction of the minima in the 


\begin{tabular}{|c|c|c|c|}
\hline \hline Eq. (6), $\Delta=0$ & Exact result, $\Delta=0$ & Eq. (6), $\Delta=0.63 \AA$ & Ref. [34] \\
\hline \hline & & & \\
56 & 58 & 56 & 58 \\
92 & 92 & 92 & 92 \\
138 & 138 & 136 & 138 \\
184 & 186 & 190 & $192 \pm 2$ \\
252 & 254 & 252 & $256 \pm 2$ \\
336 & 338 & 334 & $334 \pm 2$ \\
436 & 440 & 430 & $430 \pm 2$ \\
$540 / 554$ & $542 / 556$ & 526 & $540 \pm 5$ \\
$610 / 674$ & $612 / 676$ & 624 & $648 \pm 5$ \\
$744 / 830$ & $748 / 832$ & 752 & \\
908 & 912 & 902 & \\
1070 & 1074 & 1082 & \\
1282 & 1250 & 1286 & \\
& & & \\
\hline \hline
\end{tabular}

Table 1: Magic numbers in the perfectly spherical billiard (column 1: PO expansion, column 2 : exact results) and in the rough billiard (column 3). Column 4 shows the experimental results of Chandezon et al.

shell energy. However the electrons are sensitive to a mean-field which - due to the finite range of the electron/ion and electron/electron interaction - could be less corrugated than the ionic background. This would lead to an effective decrease of $\Delta$ and may help improving the model by reducing the importance of the ionic corrugation, leaving some room for an extra decrease in amplitude due to deformations of Jahn-Teller type.

Note that in the present treatment the effects of temperature are indirect : although the usual temperatures reached in experiments are small compared to the Fermi energy (one remains in the highly degenerate limit $k_{B} T \ll \varepsilon_{F}$ ) they are sufficient to induce a disorder of the cluster's shape which has a sizeable effect on shell structure. For comparison one can derive a formula (similar to (6)) encompassing the effect of a Fermi occupation function in the energy levels of the electron gas. The free energy $F(N, T)$ is more appropriate than the total energy for evaluating these effects. Indeed, based on Weisskopf's approach, the electronic contribution to the evaporation rate of a neutral monomer from a cluster of size $N$ can be shown to be approximatively proportional to $\exp \left\{[F(N)-F(N-1)] / k_{B} T\right\}$ [40,41]. The general formula for the oscillating part of the free energy is derived in Appendix $\mathrm{C}$ and reads in the case of a billiard :

$$
F_{\text {shell }}(N, T) \simeq \frac{\hbar^{2} \bar{k}_{\mu}^{2}}{2 m} \sum_{P O} \frac{2 \mathcal{A}\left(\bar{k}_{\mu}\right)}{\bar{k}_{\mu} L^{2}} \sin \left(\bar{k}_{\mu} L+\nu \pi / 2\right) F_{1}(\bar{X}),
$$


where $\bar{k}_{\mu}$ is the non-oscillatory part of the quantity $k_{\mu}$ defined by $\mu=\hbar^{2} k_{\mu}^{2} / 2 m, \mu$ being the chemical potential. Again $\bar{k}_{\mu}$ is to a good approximation equal to the bulk Fermi wave vector $\kappa_{F}$. $\bar{X}=(\pi / 2) \tau L \kappa_{F}^{2} / \bar{k}_{\mu}$ is a dimensionless quantity which tends to zero at $T=0\left(\tau=k_{B} T / \varepsilon_{F}\right.$ is the reduce temperature). More precisely it can be considered as small if the thermal wave length $\lambda_{T}=$ $\left(2 \pi \hbar^{2} / m k_{B} T\right)^{1 / 2}$ is large compared to $\sqrt{r_{S} L}\left(\bar{X}=2 \pi^{2} L / \bar{k}_{\mu} \lambda_{T}^{2}\right) . F_{1}$ is a dimensionless damping function defined in Appendix C (Eq. (C3)).

We compare on Fig. 3 the effects of a temperature $T=750 \mathrm{~K}$, with those of a constant roughness $\Delta=0.63 \AA$. The shell energy is displayed as a function of $N^{1 / 3}$ for sodium clusters of size $N<$ 3400. There is a striking difference with the $N$-dependence obtained via usual temperature effects on occupation numbers. As one notices from the figure, roughness damps the oscillations in the total energy with an overall factor of the type $\exp \left(-\Delta^{2} / r_{S}^{2}\right)(c f(7))$ without modifying the qualitative features of the supershells ; whereas temperature leads to a $N$-dependent damping of schematic form ( $c f$ Appendix C, Eq. (C9)) :

$$
F_{\text {shell }}(N, T) \sim \varepsilon_{F} N^{1 / 6} F_{1}\left(\tau N^{1 / 3}\right) \sin \left(N^{1 / 3}\right),
$$

(as in Eq. (7) we have omitted numerical factors in the sine and $F_{1}$ function). Hence the effects of thermal distribution of occupation numbers is to wash out the beating pattern of the shell energy by exponentially damping the large $N$ oscillations (see also Fig. 4).
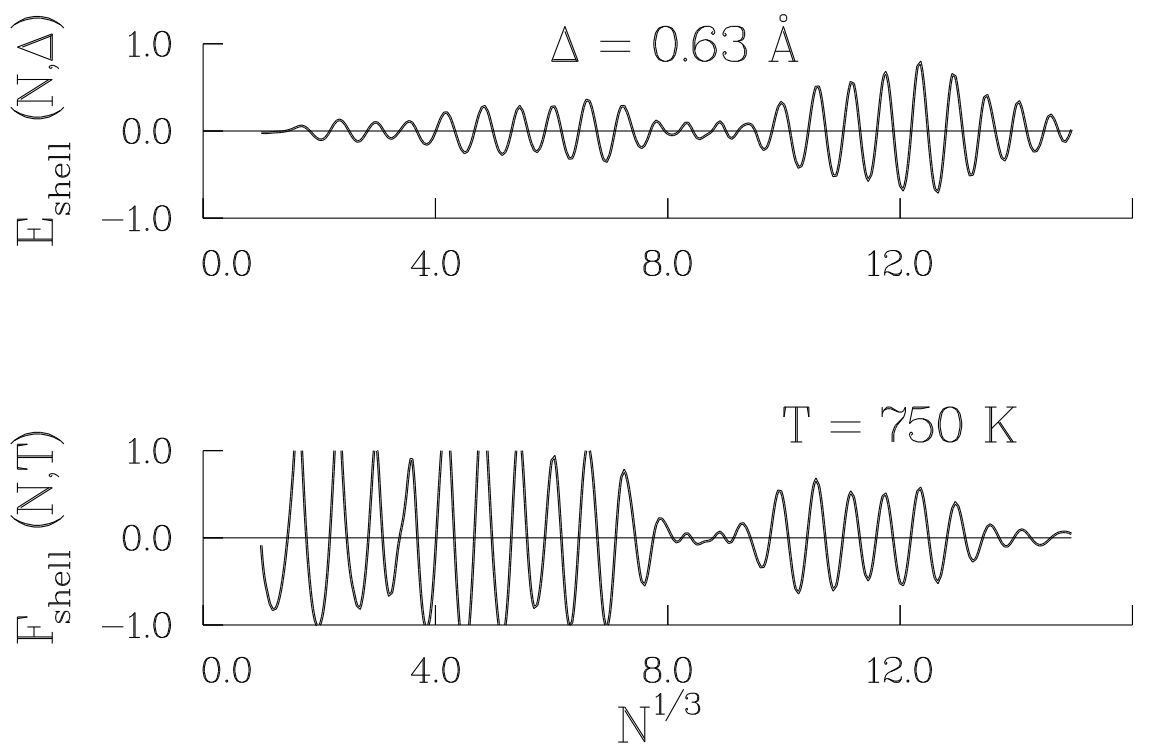

Figure 3: $E_{\text {shell }}$ (expressed in eV) as a function of $N^{1 / 3}$ in sodium clusters. The upper plot is obtained by taking a constant roughness $\Delta=0.63 \AA$. The lower plot corresponds to formula (C8) for a perfectly spherical aggregate with a temperature $\tau=k_{B} T / \varepsilon_{F}=0.02$, i.e. $T=750 \mathrm{~K}$. 
Note also the efficiency of a small roughness for diminishing shell effect : for clusters of size $800<N<1200$, from Eqs. (6) and (8), one can see for instance that the effect of a small roughness $\Delta=0.2 r_{S} \simeq R / 50$ on $E_{\text {shell }}$ is similar to the one of a temperature of about $550 \mathrm{~K}$ on $F_{\text {shell }}$. In the range $300<N<500$ the same roughness corresponds to a temperature $T \simeq 750 \mathrm{~K}$, and for $50<N<250$ it corresponds to $T \simeq 1000 \mathrm{~K}$ (around $N=200$ ) or $1400 \mathrm{~K}$ (around $N=100$ ). As a result, in a range of sizes and temperatures commonly reached in experiment $(N \sim 500$ and $T \sim 400$ $\mathrm{K})$ the effect of roughness on shell structure is dominant compared to that of thermal distribution of occupation numbers. Furthermore, as discussed above, it seems from the experimental results of Ref [34] that in about the same region, Jahn-Teller deformations of small multipolarity play a smaller role in the shape of the shell energy than predicted by usual theoretical studies (see Fig. 3 of Ref. [34]). We present here roughness as a concomitant phenomenon which (according to comparison with the data of Ref. [34]) seems more relevant for large clusters. As discussed in next section both phenomena should be taken into account for a proper description of deformations of large clusters.

\section{Discussion}

One of the original interests of metal clusters was to provide a physical realization of a discrete and random spectrum. It was long thought that the randomness of the levels would lead to a structureless level density and theoretical works were mostly devoted to the study of the two point form factor of the spectrum [42,43]. It is only during the last two decades that molecular beams have made it possible to work in a regime where the size of the cluster is well defined and small compared to the electronic mean free path. In this regime one observes shell effects as a result of finite size quantum effects [1, 迎. Nevertheless the remark of Gor'kov and Eliashberg quoted in the introduction remains valid to some extend and the present work aims to reconcile these two views by providing a semi-classical description of shell structure in presence of shape disorder.

The model we have considered is schematic ; a more elaborate procedure would be to design a Lagrangian for the surface deformation encompassing the effects of shell structure (in the spirit of Strutinsky shell corrections) :

$$
\mathcal{L}=\mathcal{L}_{L D}\left[\dot{\alpha}_{\lambda \mu}, \alpha_{\lambda \mu}\right]-E_{\text {shell }}\left[\alpha_{\lambda \mu}\right]
$$

This approach is typical in the study of deformations of finite fermionic systems. It is used for determining the equilibrium shape, i.e. the set of $\alpha_{\lambda \mu}$ minimizing the total potential energy. Between shell closure it leads to a ground-state in which the equilibrium value of some of the $\alpha$ 's is non-zero (mainly for small $\lambda$ ), contrary to what is obtained in Sec. 2 where all the $\alpha$ 's are zero at equilibrium. Using the Lagrangian (10) would also modify the stiffness of the potential near the minimum (since a term $E_{\text {shell }}$ would be added to the potential used in Sec. 2).

Following the procedure of the present paper one should go one step further and study the thermally activated vibrations of the normal modes in the Hamiltonian (10). Hence the usual Jahn-Teller deformations correspond to the first step of the procedure just exposed and to small multipolarity whereas surface roughness to the second step (and to large multipolarity).

It would be of great interest to verify if the agreement obtained in Sec. 3 with experimental values would persist when describing surface oscillations with a Lagrangian such as $\mathcal{L}$ defined in Eq. (10). This 
form of Lagrangian is legitimated by confrontation with experiments in the small and intermediate size domain, where it is commonly admitted that clusters of size $N \lesssim 100$ experience static deformations of small multipolarities [12,24]. The success of the present model (which uses $\mathcal{L}_{L D}$ and do not include such Jahn-Teller deformations) in the size range $N \gtrsim 100$ might be explained by the decrease of the shell-energy contribution to $\mathcal{L}$ due to an intrinsic roughness of the surface. Schematically one might say that there is less difference between a rough sphere and a rough ellipsoid than between a perfect sphere and a perfect ellipsoid. A similar phenomenon explains the disappearance of Jahn-Teller deformations with increasing temperature, see Ref. [40] where deformation is seen to be suppressed by thermal fluctuations. Note however that the phenomenon predicted in this reference is size-dependant, i.e. not uniform for all cluster sizes (as roughness would be), see the precise discussion in 40].

Note finally that thermally induced shape fluctuations have already been investigated in the study of the broadening of plasma resonances of metal clusters in Refs. 44 47 and very recently in the study of shell structure for clusters of size smaller than $N=100$ [39]. The ideas are similar to the one exposed above, however the allowed deformations are limited to simple shapes, whereas it has been considered necessary in the present work to include deformations of very large multipolarities for studying surface roughness.

\section{Acknowledgments}

We thank an unknown referee for pointing out the existence of Ref. [56]. We thank S. Frauendorf, J. Lermé and W. Swiatecki for fruitful discussions. We wish also to express our gratitude to S. Bjørnholm for his interest in this study, judicious remarks and inspiring comments. La Division de Physique Théorique de l'Institut de Physique Nucléaire est une unité de recherche des Universités Paris XI et Paris VI associée au CNRS.

\section{Appendix A}

In this appendix we compute the curvature energy $V_{\text {curv }}$ (defined in Eq. (30) for a droplet of shape given by (1). This amounts to evaluate the integral $\mathcal{C}=\int d A\left(1 / \mathcal{R}_{1}+1 / \mathcal{R}_{2}\right)$ for a boundary approximatively spherical. This can be done by noticing that if $\mathcal{A}$ is the surface area of a given boundary, the modification $\delta \mathcal{A}$ caused by an infinitesimal displacement of the boundary reads $\delta \mathcal{A}=\int d A \delta \zeta\left(1 / \mathcal{R}_{1}+\right.$ $1 / \mathcal{R}_{2}$ ), where $\delta \zeta$ is the normal segment between the undeformed boundary and the deformed one (see eg. [48], chap. VII). If this modification corresponds to a modification of Eq. (1]) by $r \rightarrow r+\delta r$, one can compute $\delta \mathcal{A}$ and $\delta \zeta$ in terms of $\delta r$. This allows to write $\mathcal{C}$ in the form

$$
\mathcal{C}=\int d \Omega \frac{K}{r}\left\{\frac{r^{2}+K}{K^{1 / 2}}-\frac{1}{\sin \theta} \partial_{\theta}\left(\frac{r \sin \theta \partial_{\theta} r}{K^{1 / 2}}\right)-\frac{1}{\sin ^{2} \theta} \partial_{\phi}\left(\frac{r \partial_{\phi} r}{K^{1 / 2}}\right)\right\}
$$

where $K(\Omega)=r^{2}+\left(\partial_{\theta} r\right)^{2}+\left(\partial_{\phi} r\right)^{2} / \sin ^{2} \theta$. Then, writing $r(\Omega)=R[1+h(\Omega)]$ and neglecting terms of order greater than $\mathcal{O}\left(h^{2}\right)$ one obtains :

$$
\mathcal{C}=2 R \int d \Omega\left\{1+h+\frac{1}{2}\left(\partial_{\theta} h\right)^{2}+\frac{1}{2 \sin ^{2} \theta}\left(\partial_{\phi} h\right)^{2}\right\}+\mathcal{O}\left(h^{3}\right)
$$

The surface area can be expressed in a similar manner (see [48) : 


$$
\mathcal{A}=\int d A=\int d \Omega r K^{1 / 2}=R^{2} \int d \Omega\left\{(1+h)^{2}+\frac{1}{2}\left(\partial_{\theta} h\right)^{2}+\frac{1}{2 \sin ^{2} \theta}\left(\partial_{\phi} h\right)^{2}\right\}+\mathcal{O}\left(h^{3}\right) .
$$

The condition of volume conservation imposes $\int d \Omega(1+h)^{2}=\int d \Omega(1+h)+\mathcal{O}\left(h^{3}\right)$. Hence, comparing Eqs. (A2) and (A3) one sees that for small deformations the curvature integral is proportional to the surface area : $\mathcal{C}=2 \mathcal{A} / R+\mathcal{O}\left(h^{3}\right)$. The corrections are of third order in the deformation, they are given in Ref. [49] (chap. 6) for spheroidal and harmonic deformations. Here we are interested only in terms up to order $\mathcal{O}\left(h^{2}\right)$, thus the curvature energy $V_{\text {curv }}=\gamma \mathcal{C} / 4$ is equal up to a multiplicative constant to the surface term $V_{\text {surf }}=\sigma \mathcal{A}: V_{\text {curv }}$ is formally obtained from $V_{\text {surf }}$ by replacing the surface tension $\sigma$ by an effective term $\gamma /(2 R)$.

\section{Appendix B}

In this appendix we briefly present the results of Ref. [33] for the level density in the sphere and we make explicit the different terms appearing in Eq. (6) for the perfect billiard.

The periodic orbits in the sphere are regular polygons in diametral planes. They are labeled by two numbers $(n, t), n$ being the number of sides and $t$ the winding number of the orbit around the center $(n \geq 2 t)$. Note that $n$ is here the same as the number of bouncing points appearing in the main text (Eq. (6)). The oscillating part of the level density in the sphere reads :

$$
\rho_{\mathrm{osc}}(k)=\sum_{t=1}^{+\infty} \sum_{n=2 t}^{+\infty} \mathcal{A}_{n, t}(k) \sin \left(k L_{n, t}+\nu_{n, t} \pi / 2\right)
$$

The shortest orbits are the pendulating orbit $(n=2, t=1)$, the triangle $(n=3, t=1)$, and the square $(n=4, t=1)$. The triangle and the square are sufficient to understand the qualitative features of the shell and supershell structure (see e.g. [50,51]). Each orbit bounces on the surface with a constant normal angle $\theta_{n, t}=(1-2 t / n) \pi / 2$ and has a length $L_{n, t}=2 n R \cos \theta_{n, t}$. The pendulating orbit occurs in a two-parameters family (the parameters determine the direction of bouncing) whereas all the other orbits form three-parameters families. Hence the bouncing ball (with $n=2 t$ ) has to be treated separately. The explicit formulae for $\mathcal{A}(k)$ and $\nu$ in Eqs. (6) and (B1) are $(t \geq 1)$ :

$$
\nu_{n, t}=\left\{\begin{array}{ccc}
0 & \text { if } & n=2 t \\
n+3 / 2 & \text { if } & n>2 t
\end{array}\right.
$$

and

$$
\mathcal{A}_{n, t}(k)=\left\{\begin{array}{lll}
-\frac{d_{S} k R^{2}}{\pi t} & \text { if } & n=2 t, \\
2 d_{S}(-1)^{t} \sin \left(2 \theta_{n, t}\right) \sqrt{\frac{\cos \theta_{n, t}}{\pi n}} R(R k)^{3 / 2} & \text { if } & n>2 t
\end{array}\right.
$$

where $d_{S}=2$ is the spin degeneracy.

One sees that the amplitude corresponding to the pendulating orbit is proportional to $k$ whereas the other families have a larger weight (proportional to $k^{3 / 2}$ ). Generally speaking, one can show that the contribution of a $d$-parameter family has an extra $k^{d / 2}$ power with respect to that of an isolated orbit 52]. 


\section{Appendix C}

In this appendix we derive approximate analytical expressions for the oscillatory part of the total energy and of the free energy at finite temperature. Similar results concerning the entropy, the free energy etc... have been previously obtained by Kolomiets, Magner and Strutinsky in Refs. [53, 54]. We nevertheless briefly outline the derivation of the formulae because the references just quoted are not very explicit and difficult to follow. The formulae are derived in the framework of a general PO expansion : the level density is noted $\rho(\epsilon)$, it is separated in a smooth term $\bar{\rho}(\epsilon)$ and an oscillating term $\rho_{\text {osc }}(\epsilon)$. In the present work, we denote all the smooth terms with an upper bar and the oscillating terms with a subscript "osc", except for the oscillating part of the energies which have a subscript "shell" according to the general convention in the field. $\rho_{\text {osc }}(\epsilon)$ is supposed to be of the form :

$$
\rho_{\text {osc }}(\epsilon)=\operatorname{Re} \sum_{P O} \mathcal{B}(\epsilon) \mathrm{e}^{i S(\epsilon) / \hbar}
$$

where $S(\epsilon)$ is the action of the PO considered. $\mathcal{B}(\epsilon)$ is an orbit dependent amplitude which is of order $\hbar^{-1}$ for chaotic systems, of order $\hbar^{-2}$ for typical integrable systems in three dimensions and of order $\hbar^{-5 / 2}$ for rotationally symmetric systems as the spherical billiard where families of orbits are characterized by 3 parameters [52].

We will estimate the asymptotic form of several integrals, all of the same type, and we first display a formula often used below. Let $g(\epsilon)$ be a slowly varying function of $\epsilon$ (as $\mathcal{B}(\epsilon)$ is supposed to be), and $g^{\prime}$ its first derivative. Let $\phi(\epsilon-\mu)=\left[1+\exp \left\{(\epsilon-\mu) / k_{B} T\right\}\right]^{-1}$ be the Fermi function, $\mu$ being the chemical potential. If $S(\mu) \gg \hbar$ one has :

$$
\int_{0}^{+\infty} g(\epsilon) \phi(\epsilon-\mu) \mathrm{e}^{i S(\epsilon) / \hbar} d \epsilon=\frac{\hbar}{i} \frac{\mathrm{e}^{i S(\mu) / \hbar}}{S^{\prime}(\mu)}\left\{g(\mu) F_{1}(X)-\frac{\hbar}{i} \frac{g^{\prime}(\mu)}{S^{\prime}(\mu)} F_{2}(X)+\frac{\hbar}{i} \frac{g(\mu) S^{\prime \prime}(\mu)}{\left[S^{\prime}(\mu)\right]^{2}} F_{3}(X)+\cdots\right\},
$$

where the integral has been evaluated by a contour integration in the complex plane (see e.g. [55]). In the evaluation of the integral we have neglected the contribution of a part of the contour located on the positive imaginary axis, this is legitimate provided the temperature is small compared to the Fermi energy (degenerate Fermi gas approximation). $X=\pi S^{\prime}(\mu) k_{B} T / \hbar$ is a dimensionless quantity which can be considered as small if the period $S^{\prime}$ of the orbit is small compared to a characteristic thermal time $\hbar / k_{B} T . F_{1}, F_{2}$ and $F_{3}$ are dimensionless damping functions :

$$
F_{1}(X)=\frac{X}{\sinh X}, \quad F_{2}(X)=\frac{X^{2} \cosh X}{\sinh ^{2} X}, \quad F_{3}(X)=\frac{X^{3}}{\sinh ^{3} X}\left(1+\frac{\sinh ^{2} X}{2}\right) .
$$

For obtaining the total energy starting from (C1), one first determines the chemical potential $\mu$ through the equality $N=\mathcal{N}(\mu)$, where $N$ is the number of electrons and $\mathcal{N}(\mu)=\int \rho(\epsilon) \phi(\epsilon-\mu) d \epsilon$. As $\rho(\epsilon), \mathcal{N}$ can be separated in a smooth term $\overline{\mathcal{N}}$ (the Weyl term) plus an oscillating part $\mathcal{N}_{\text {osc }}$. Accordingly, $\mu$ can be separated in a smooth function of $N$ plus an oscillating term $: \mu=\bar{\mu}+\mu_{\mathrm{osc}}$, where $N=\overline{\mathcal{N}}(\bar{\mu})$.

Then the total electronic energy is $E=\int \epsilon \phi(\epsilon-\mu) \rho(\epsilon) d \epsilon$. It can also be separated into a smooth part $\bar{E}$ and an oscillating part which is denoted $E_{\text {shell }}$ throughout the paper (more precisely $E_{\text {shell }}(N, T)$ 
in presence of temperature) in accordance with the general notations in the field. $E_{\text {shell }}$ reads approximatively :

$$
\begin{aligned}
E_{\text {shell }} & =\int \epsilon \rho(\epsilon) \phi(\epsilon-\mu) d \epsilon-\int \epsilon \bar{\rho}(\epsilon) \phi(\epsilon-\bar{\mu}) d \epsilon \\
& \simeq \int \epsilon \rho(\epsilon)\left[\phi(\epsilon-\bar{\mu})-\mu_{\mathrm{osc}} \phi^{\prime}(\epsilon-\bar{\mu})\right] d \epsilon-\int \epsilon \bar{\rho}(\epsilon) \phi(\epsilon-\bar{\mu}) d \epsilon \\
& =\int \epsilon \rho_{\mathrm{osc}}(\epsilon) \phi(\epsilon-\bar{\mu}) d \epsilon+\mu_{\mathrm{osc}} \bar{\mu}\left(\frac{d \mathcal{N}}{d \mu}\right)_{\bar{\mu}}-\mu_{\mathrm{osc}} \int(\epsilon-\bar{\mu}) \rho(\epsilon) \phi^{\prime}(\epsilon-\bar{\mu}) d \epsilon .
\end{aligned}
$$

The last term of the r.h.s. of $(\overline{\mathrm{C} 4})$ is subdominant, moreover it is zero at zero temperature, hence we drop it in the following. Then, from (C1) and (C2) one obtains :

$$
E_{\text {shell }}(N, T) \simeq-\operatorname{Re} \sum_{P O}\left(\frac{\hbar}{i S^{\prime}(\bar{\mu})}\right)^{2} \mathcal{B}(\bar{\mu}) F_{2}(\bar{X}) \mathrm{e}^{i S(\bar{\mu}) / \hbar},
$$

where $\bar{X}$ is computed as $X$ with $\bar{\mu}$ replacing $\mu$.

The free energy $F(N, T)$ is a quantity more appropriate than the total energy to evaluate the effects of electronic temperature on the abundance of clusters in the beam (see the discussion in the main text). It is defined by

$$
F(N, T)=\mu N+\int_{0}^{+\infty} d \epsilon \rho(\epsilon) \Phi(\epsilon-\mu) \quad \text { where } \quad \Phi(\epsilon-\mu)=-k_{B} T \ln \left(1+\mathrm{e}^{(\mu-\epsilon) / k_{B} T}\right) .
$$

The oscillating part of the free energy is denoted by $F_{\text {shell }}(N, T)$ and can be evaluated similarly to what has been done in (C4). This yields :

$$
F_{\text {shell }}(N, T) \simeq-\operatorname{Re} \sum_{P O}\left(\frac{\hbar}{i S^{\prime}(\bar{\mu})}\right)^{2} \mathcal{B}(\bar{\mu}) F_{1}(\bar{X}) \mathrm{e}^{i S(\bar{\mu}) / \hbar},
$$

In the particular case of a billiard whose level density is of the type (B1), Eq. (C7) reads :

$$
F_{\text {shell }} \simeq \frac{\hbar^{2} \bar{k}_{\mu}^{2}}{2 m} \sum_{P O} \frac{2 \mathcal{A}\left(\bar{k}_{\mu}\right)}{\bar{k}_{\mu} L^{2}} \sin \left(\bar{k}_{\mu} L+\nu \pi / 2\right) F_{1}(\bar{X}),
$$

where $k_{\mu}$ is defined by $\mu=\hbar^{2} k_{\mu}^{2} /(2 m)$. A formula of this type seems to have been derived first by Dingle in Ref. [56]. We have verified that this formula is of very good accuracy in the spherical billiard (see Fig. 4). An equally good agreement is obtained for the comparison of $E_{\text {shell }}$ (as given by (C5)) with the exact result. For relatively low values of $N^{1 / 3}$ (say $N^{1 / 3}<6$ ), an even better agreement can be obtained by still using the semi-classical level density, but evaluating integrals such as (C6) numerically.

In the sphere, the main contribution to (C8) comes from orbits occurring in three-parameters families (cf Appendix B). Considering that $\bar{k}_{\mu}$ is of order $\kappa_{F} \sim 1 / r_{S}$ and that $L$ and $R$ scale like $r_{S} N^{1 / 3}$ one obtains the following leading order : $\mathcal{A}\left(\bar{k}_{\mu}\right) \sim R\left(R \bar{k}_{\mu}\right)^{3 / 2} \sim r_{S} N^{5 / 6}$. Hence the schematic large $N$ behaviour of (C8) reads 

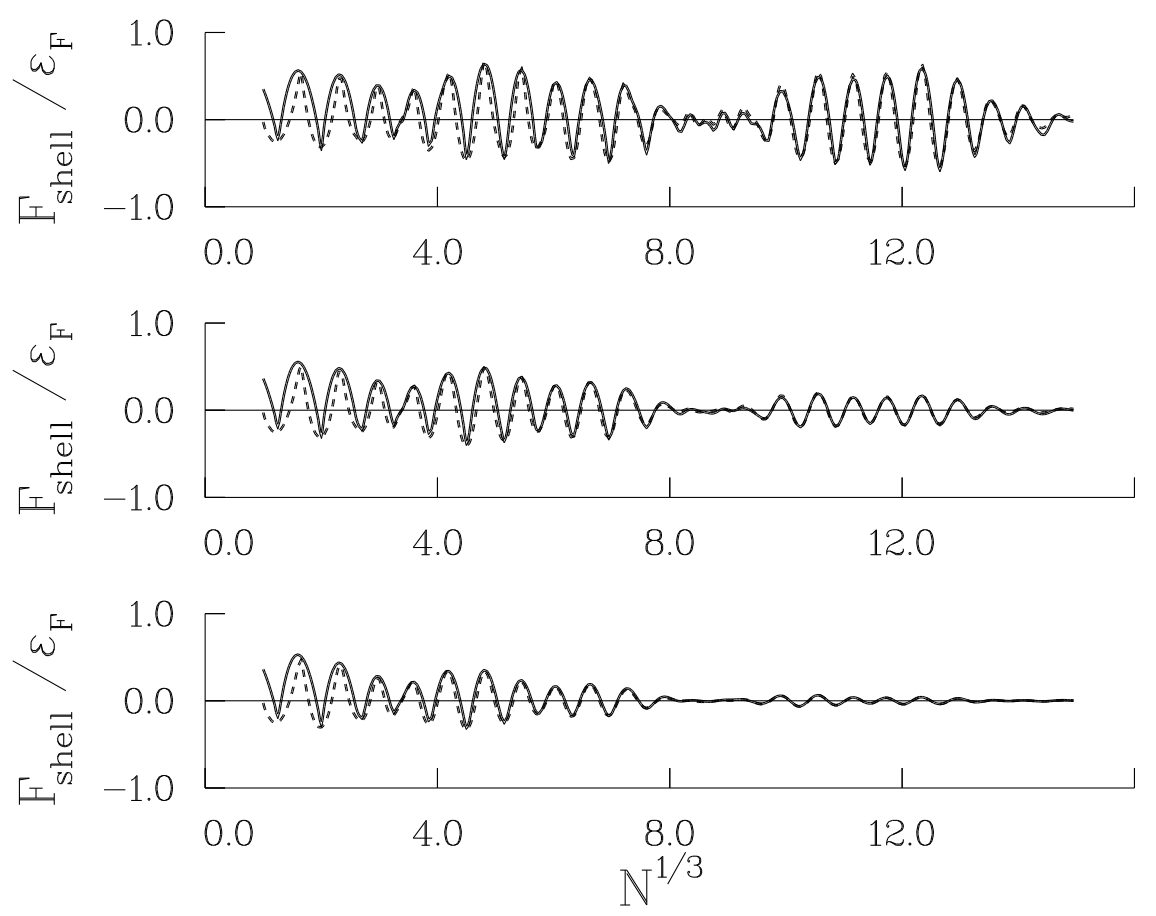

Figure 4: $F_{\text {shell }}$ (expressed in units of the bulk Fermi energy $\varepsilon_{F}$ ) as a function of $N^{1 / 3}$ in a perfectly spherical aggregate. $F_{\text {shell }}$ is denoted $F_{\text {shell }}(N, T)$ in the main text. The different plots correspond to temperatures $\tau=0.01$ (upper plot), $\tau=0.02$ and 0.03 (lower plots), where $\tau=k_{B} T / \varepsilon_{F}$. For sodium these values correspond to $T=376 \mathrm{~K}, 752 \mathrm{~K}$ and $1128 \mathrm{~K}$. The solid lines correspond to the determination of $F_{\text {shell }}$ obtained by using the exact spectrum of the spherical billiard, and the dashed lines to Eq. (C8).

$$
F_{\text {shell }} \sim \varepsilon_{F} N^{1 / 6} F_{1}\left(\tau N^{1 / 3}\right) \sin \left(N^{1 / 3}\right) .
$$

where $\tau=k_{B} T / \varepsilon_{F}$ is the temperature expressed in units of the bulk Fermi energy. Here we have dropped for clarity important but dimensionless factors in the sine and $F_{1}$ function : we just want to illustrate the typical $N$ dependence of $F_{\text {shell }}$. We have adopted the same type of notation in the text (0.9). The behaviour ( $\mathrm{C9}$ ) is in agreement with the findings of Kolomiets, Magner and Strutinsky in Refs. [53, 54 and with Ref. [18] where Bohr and Mottelson used schematic forms of the level density. We emphasize that we have used here a generic PO expansion, and Eqs. (C5,C7) are valid for any system (chaotic or integrable) of independent fermions moving in an external potential.

Note finally that we have here computed the thermodynamical quantities in the grand canonical ensemble. The number of electrons in a cluster being exactly conserved, the canonical description should be used instead (hence we should have noted $F(\mu, T)$ instead of $F(N, T)$ : in all the appendix 
$N$ should be understood as the mean number of electrons). The difference between the two ensembles has been studied in Ref. [57] where it is shown to give discrepancies of order of $0.05 \mathrm{eV}$ (or $0.1 \mathrm{eV}$ at best) in the free energy difference $F(N-1)-F(N)$. This difference is expected to decrease in the

large $N$ limit and moreover it plays no role in the discussion of the effects of temperature given in the main text.

\section{References}

[1] M. Brack, Rev. Mod. Phys. 65, 677 (1993).

[2] U. Näher, S. Bjørnholm, S. Frauendorf, F. Garcias and C. Guet, Phys. Rep. 285, 245 (1997).

[3] N. D. Lang, in Solid State Physics edited by H. Ehrenreich and D. Turnbull, Vol. 28 (Academic, New-York, 1973).

[4] W. A. de Heer, Rev. Mod. Phys. 65, 611 (1993).

[5] W. Ekardt and Z. Penzar, Phys. Rev. B 38, 4273 (1988).

[6] T. Hirschmann, M. Brack and J. Meyer, Ann. Physik 3, 336 (1994), ibidem Comp. Mat. Sci. 2, 450 (1994).

[7] M. Koskinen, P. O. Lipas and M. Manninen, Europhys. Lett. 30, 519 (1995).

[8] K. Clemenger, Phys. Rev. B 32, 1359 (1985).

[9] S. M. Reimann, M. Brack, Klavs Hansen, Z. Phys. D 28, 235 (1993).

[10] A. Bulgac and C. Lewenkopf, Phys. Rev. Lett. 71, 4130 (1993).

[11] S. M. Reimann and M. Brack, Comp. Mat. Sci. 2, 433 (1994).

[12] C. Yannouleas and U. Landman, Phys. Rev. B 51, 1902 (1995).

[13] S. Frauendorf and V. V. Pashkevich, Ann. Physik 5, 34 (1996).

[14] L. P. Gor'kov and G. M. Eliashberg, Zh. Eksp. Fiz. 48, 1407 (1965) ; Sov. Phys. JETP 21, 940 (1965).

[15] H. Nishioka, Klavs Hansen and B. R. Mottelson, Phys. Rev. B 42, 9377 (1990).

[16] N. Pavloff, J. Phys. A 28, 4123 (1995).

[17] Lord Rayleigh, The Theory of Sound, Vol. II, Dover, New-York, 1945 (reprinted from MacMillan, New-York, 1896).

[18] A. Bohr and B. R. Mottelson, Nuclear Structure, Vol. II (Benjamin, New-York, 1975).

[19] W. R. Tyson and W. A. Miller, Surf. Sci. 62, 267 (1977).

[20] J. P. Perdew, Y. Wand and E. Engel, Phys. Rev. Lett. 66, 508 (1991). 
[21] M. W. Cole, Phys. Rev. A 1, 1838 (1970).

[22] M. Brack, Phys. Rev. B 39, 3533 (1989).

[23] C. Bréchignac, H. Busch, Ph. Cahuzac and J. Leygnier, J. Chem. Phys. 101, 6992 (1994).

[24] C. Yannouleas and U. Landman, J. Chem. Phys. 105, 8734 (1996) ; J. Chem. Phys. 107, 1032 (1997).

[25] K. F. Ratcliff in Multivariate Analysis V, page 593, P. R. Krishnaiah ed. (North-Holland, Amsterdam, 1980).

[26] J. P. Bucher, P. Xia and L. A. Bloomfield, Phys. Rev. B 42, 10858 (1990).

[27] N. Pavloff and M. S. Hansen, Z. Phys. D 24, 57 (1992).

[28] J. Mansikka-aho, M. Manninen and E. Hammarén, Phys. Rev. B 47, 10675 (1993).

[29] K. M. Frahm and D. L. Shepelyansky, Phys. Rev. Lett. 78, 1440 (1997).

[30] C. Bréchignac, Ph. Cahuzac, J. Leygnier, A. Sarfati and V. M. Akulin, Phys. Rev. A 51, 3902 (1995).

[31] V. M. Akulin, C. Bréchignac and A. Sarfati, Phys. Rev. Lett. 75, 220 (1995).

[32] J. Lermé, M. Pellarin, E. Cottancin, B. Baguenard, J. L. Vialle and M. Broyer, Phys. Rev. B 52, 14163 (1995).

[33] R. Balian and C. Bloch, Ann. Phys. 69, 76 (1972).

[34] F. Chandezon, S. Bjørnholm, J. Borggreen and K. Hansen, Phys. Rev. B 55, 5485 (1997).

[35] J. Leygnier, Ph.D. Thesis, Université de Paris Sud, Centre d'Orsay, 1989.

[36] C. Bréchignac, Ph. Cahuzac, J. Leygnier and J. Weiner, J. Chem. Phys. 90, 1492 (1989).

[37] K. Hansen and J. Falk, Z. Phys. D 34, 251 (1995).

[38] N. Pavloff and C. Schmit, in preparation.

[39] C. Yannouleas and U. Landman, Phys. Rev. Lett. 97, 1424 (1997).

[40] S. Frauendorf and V. V. Pashkevich, in Large Clusters of Atoms and Molecules, Vol. 313 of NATO advanced studies institute series E; applied sciences, T. P. Martin ed. (Kluwer Academic, Dordrecht, 1996),p 201.

[41] K. Hansen and M. Manninen, J. Chem. Phys. 101, 10481 (1994).

[42] J. A. A. J. Perenboom, P. Wyder, F. Meier, Phys. Rep. C78, 173 (1981).

[43] W. P. Halperin, Rev. Mod. Phys. 58, 533 (1986). 
[44] G. F. Bertsch and D. Tománek, Phys. Rev. B 40, 2749 (1989).

[45] J. M. Pacheco and R. A. Broglia, Phys. Rev. Lett. 62, 1400 (1989).

[46] C. Yannouleas, J. M. Pacheco and R. A. Broglia, Phys. Rev. B 41, 6088 (1990).

[47] Z. Penzar, W. Ekardt and A. Rubio, Phys. Rev. B 42, 5040 (1990).

[48] L. D. Landau and E. M. Lifshitz, Course of Theoretical Physics, Vol. 6, Fluid Mechanics, (Pergamon Press, Oxford, 1980).

[49] R. W. Hasse and W. D. Myers, "Geometrical Relationships of Macroscopic Nuclear Physics", (Springer-Verlag, Berlin, 1988).

[50] J. Pedersen, S. Bjørnholm, J. Borggreen, K. Hansen, T. P. Martin and H. D. Rasmussen, Nature 353, 733 (1991).

[51] P. Sampfli and K. H. Bennemann, Z. Phys. D 25, 87 (1992).

[52] S. C. Creagh and R. G. Littlejohn, Phys. Rev. A 44, 836 (1991) ; J. Phys. A 25, 1643 (1992).

[53] V. M. Strutinsky and A. G. Magner, Sov. J. Part. Nucl. 7, 138 (1976).

[54] V. M. Kolomiets, A. G. Magner and V. M. Strutinsky, Sov. J. Nucl. Phys. 29, 758 (1979).

[55] K. Richter, D. Ullmo and R. A. Jalabert, Phys. Rep. 276, 1 (1996).

[56] R. B. Dingle, Proc. Roy. Soc. A, 212, 47 (1952).

[57] M. Brack, O. Genzken and K. Hansen, Z. Phys. D 21, 65 (1991). 